\title{
Study on the Performance of the Physical Foaming Warm-mix Recycled Asphalt Mixture
}

\author{
Zheng Zhigang ${ }^{1}$, He Yunwu ${ }^{2}$, Wang Tao ${ }^{1}$, Wei Hanxin ${ }^{1}$, Liang Xiayi ${ }^{1}, \mathrm{Xiao}_{\mathrm{Xin}}^{3}$, Liu Tao ${ }^{1 *}$ \\ ${ }^{1}$ Shenzhen Yuetong Construction Engineering Co., Ltd, 518019, Shenzhen, China \\ ${ }^{2}$ Shenzhen Tagen<group $>$ Co., Ltd, 518034, Shenzhen, China \\ ${ }^{3}$ Foshan University, 528000, Foshan, China
}

\begin{abstract}
In order to evaluate the performance of the physical foaming warm-mix recycled asphalt mixture (WRAM), the hot mix plant recycling technology was adopted to develop AC-20 hot-mix recycled asphalt mixture (HRAM) and the WRAM of different reclaimed asphalt pavement (RAP) contents. The high temperature stability, water stability and low temperature crack resistance performance of the reclaimed asphalt pavement were evaluated. The results indicate that the physical foaming WRAM feature better high temperature stability, water stability and low temperature crack resistance compared to those of HRAM. With the increase in RAP contents, the high temperature stability of the physical foaming WRAM and HRAM rises notably but the water stability and low temperature crack resistance performance continues to drop.
\end{abstract}

\section{Introduction}

With the development of China's economy, the highway construction has gradually shifted from "construction oriented" to "equal attention paid to construction and maintenance". Considerable asphalt mixture waste have been generated from large-scale asphalt pavement maintenance, most of which are piled up, occupying land and polluting the environment [1-2]. If the original asphalt pavement can be recycled to restore the performance of the aging asphalt and be re-paved on the road surface, it can not only save resources but also create substantial economic benefits and environmental protection effects. The hot mix plant recycling technology is the most-used road surface recycling technology at home and abroad. However, due to the high production temperature, the RAP will further age after reheating and a large amount of hazardous gas and asphalt fumes will be generated during the production [3-5]. Given this, some scholars have proposed to combine the recycling technology and the warm mix technology to develop the warm-mix recycling technology. This technology has both the advantages of warm mix and the recycling technology, which can effectively increase the mix ratio of RAP while reducing the production temperature [3-8].

Existing researches on the warm-mix recycling asphalt technology mainly focus on the WRAM with addition of the warm mix agent [9-10]. Few research have been carried out on the physical foaming warm-mix asphalt mixture. Considering this, this paper took AC-20 as an example to study the performance of the physical foaming warm-mix recycled asphalt mixture and studied the influence of different RAP contents on the road performance using HRAM and WRAM. Hopefully, it can provide guidance for the promotion and application of the physical foaming warm-mix recycling technology.

\section{Asphalt foaming performance}

Foamed asphalt uses the expansion ratio and the half-life period to evaluate the foaming performance of the asphalt. In this paper, the best foaming effect is obtained by changing the expansion ratio and half-life. Both the expansion ratio and the half-life should be as large as possible to get a good foaming effect. The foaming test is carried out under the conditions of asphalt temperature of $135^{\circ} \mathrm{C}, 145^{\circ} \mathrm{C}$ and $155^{\circ} \mathrm{C}$, and foaming water consumption of $1 \%, 1.5 \%, 2.0 \%, 2.5 \%, 3.0 \%, 3.5 \%$ and $4.0 \%$ respectively to measure the expansion ratio and the half-life period. The test results are shown in Fig. 1.

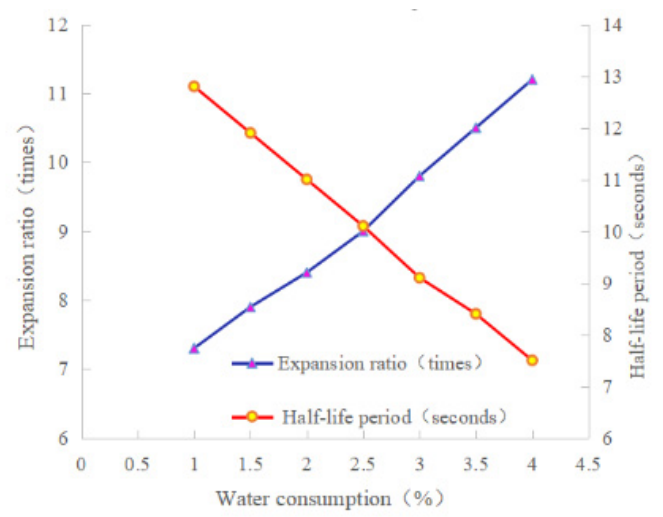

(a) $135^{\circ} \mathrm{C}$

*Corresponding author: 315778102@qq.com 


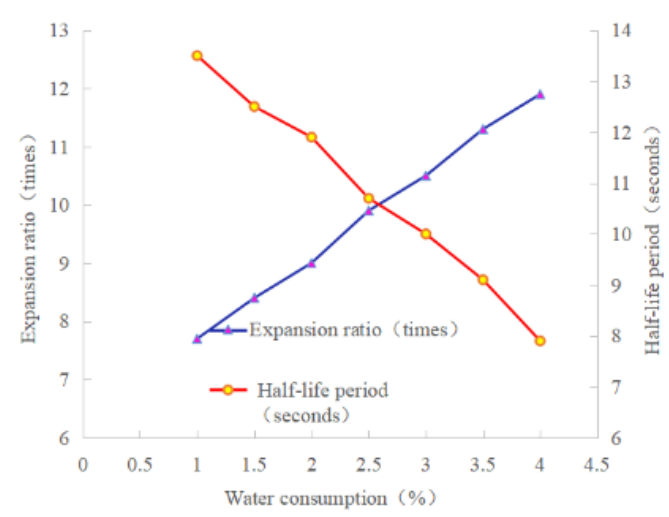

(b) $145^{\circ} \mathrm{C}$

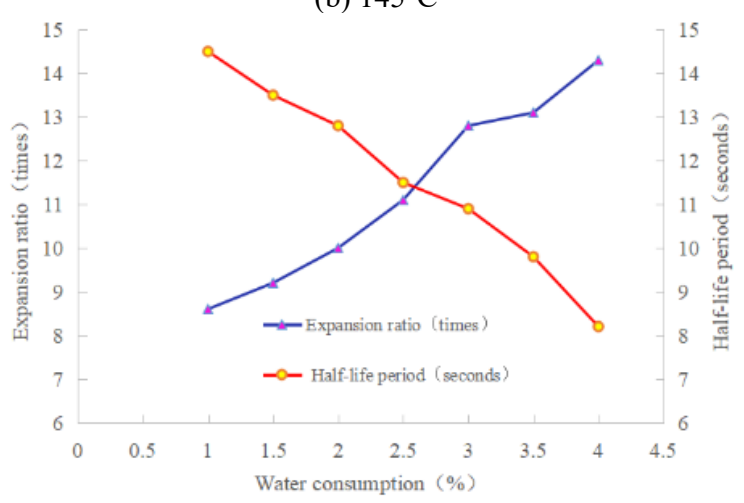

(c) $145^{\circ} \mathrm{C}$

Fig. 1 Asphalt foaming test results

According to the test results shown in Fig. 1, when the water consumption is about $2.5 \%$, the expansion ratios and half-life periods at a temperature of $135^{\circ} \mathrm{C}, 145^{\circ} \mathrm{C}$, and $155^{\circ} \mathrm{C}$ are 9 times and $10.1 \mathrm{~s}, 9.9$ times and $10.7 \mathrm{~s}, 11.0$ times and $11.6 \mathrm{~s}$, respectively. It can be seen that conditional on this water consumption, the expansion ratio and the half-life period at these three temperatures can achieve good values and the two can reach a good balance. Accordingly, the optimal water consumption of the foamed asphalt is $2.5 \%$. The foaming temperature was set at a temperature of $145^{\circ} \mathrm{C}$ with reference to warm-mix asphalt construction experience and related research.

\section{Reclaimed asphalt pavement ratio design}

The foamed asphalt is produced by adding a minimum of water to hot asphalt so the asphalt can produce tiny bubbles and expand. The foaming process is mainly a physical change, which doesn't affect the production of the HRAM. Therefore the ordinary hot mix plant recycling technology can be used for design.

The RAP contents used in this paper were $30 \%, 40 \%$, and $50 \%$. The AC-20 asphalt mixture was selected as the research object to explore the impact of the RAP content on the WRAM performance. Based on the above foaming characteristics study, the asphalt pavement mixing temperature and compaction temperature were determined as $145^{\circ} \mathrm{C}$ and $135^{\circ} \mathrm{C}$, respectively.

\subsection{RAP performance evaluation}

In this paper, RAP materials were selected from municipal roads that had been open to traffic for more than 10 years, and were obtained by milling the $5 \mathrm{~cm}$ middle layer of the original road surface. The RAP aggregates present good performance.

\subsection{Aggregates}

Both the coarse and the fine aggregates used in this paper are granites. A basic physical performance test was carried out, all satisfying the specification requirements.

\subsection{Asphalt}

The base asphalt used in this paper is the No. 70 A-grade road petroleum asphalt, the foaming temperature $145^{\circ} \mathrm{C}$, and the foaming water consumption $2.5 \%$. The performance of the asphalt before and after foaming were tested and tests results all satisfying the specification requirements.

\subsection{Mixture design}

The RAP contents used in this paper were $30 \%, 40 \%$, and $50 \%$. The design gradation was AC-20.

Based on related engineering experience: the best asphalt aggregate ratio of AC-20 type HRAM when the RAP content is $40 \%$ is $4.5 \%$. Thus, this paper utilized 5 asphalt aggregate ratios of $3.5 \%, 4.0 \%, 4.5 \%, 5.0 \%$ and $5.5 \%$ to prepare HRAM. The Marshall compaction method was used to form the asphalt mixture test piece to test the volume indicator. Finally it was determined that the best asphalt aggregate ratio of HRAM with $40 \%$ RAP is $4.1 \%$. The same method was adopted to determine the best asphalt aggregate ratios of the HRAM with $30 \%$ and $50 \%$ RAP is $4.3 \%$ and $4.1 \%$, respectively.

The gradation and the best asphalt aggregate ratio of the physical foaming WRAM is identical to those of the HRAM.

\section{Study on the performance of recycled asphalt mixture}

\subsection{High temperature stability}

The test results are shown in Fig. 2.

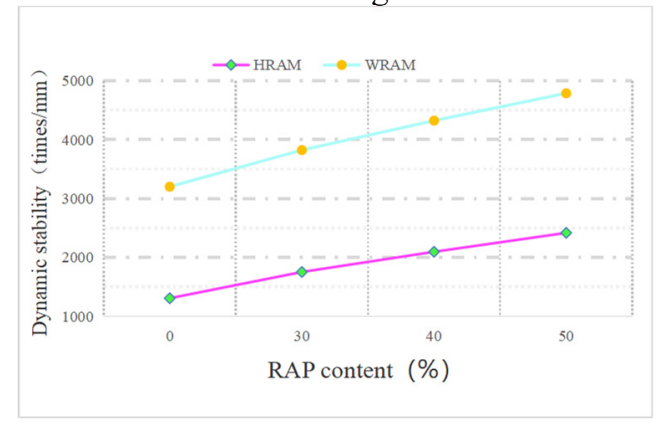

Fig.2 High temperature stability test results 
It can be drawn from Fig. 2 that the dynamic stability of the WRAM and the HRAM both satisfies the standard requirements: conditional on the same RAP content, the dynamic stability of the WRAM is higher than that of the HRAM, which is more than doubled, and the growth rate increases slightly with the increase in the RAP contents. This is mainly due to the fact that the physical foaming asphalt mixtures were mixed at a lower production temperature, which reduces the short-term aging of the asphalt. Regardless of whether it is the WRAM or the HRAM, its dynamic stability increases significantly with the increase of the RAP content, which indicates that the addition of the RAP can notably improve the high temperature stability of the recycled asphalt mixtures, and the two are in a positive correlation.

\subsection{Water stability}

The test results are shown in Fig. 3.
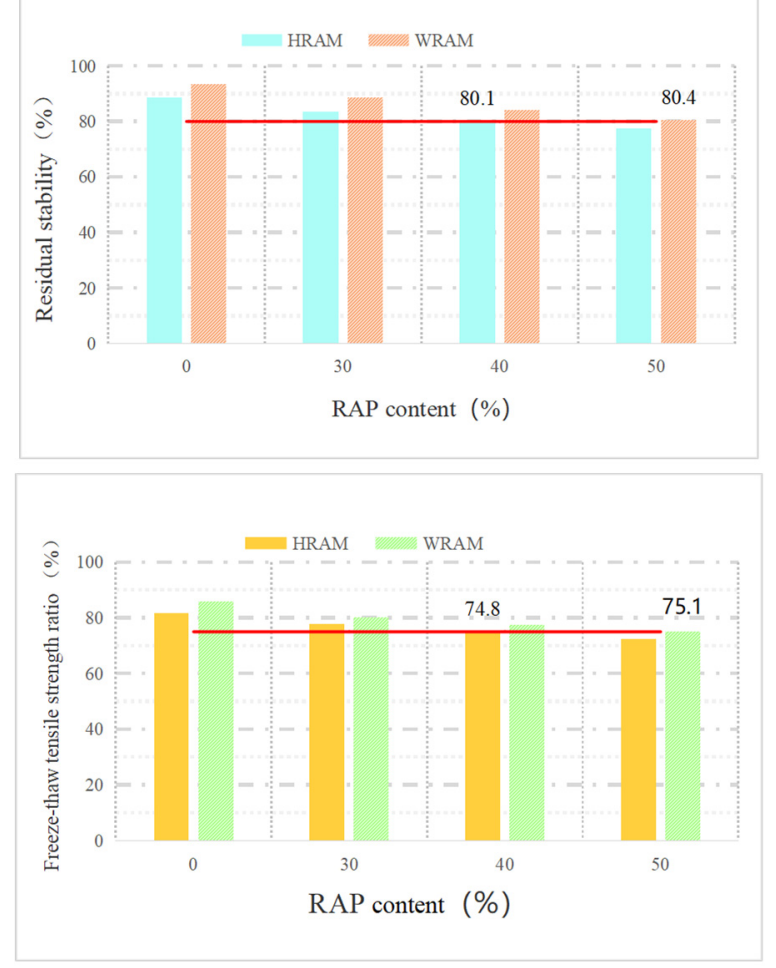

Fig. 3 Water stability of the asphalt mixture

1). It can be seen from Fig. 3 that both the retained Marshall stability and the freeze-thaw tensile strength ratio of the WRAM and the HRAM drop as the RAP content increases, indicating that the water stability performance of the recycled asphalt mixture is worse than that of the new-mix asphalt mixture.

2). Conditional on the same RAP content, WRAM features better water stability than HRAM, which is due to the high adhesion of the foamed asphalt and the aggregates. As a result, the peeling resistance of the asphalt mixture is enhanced and then it shows good water stability.

3). When the RAP content reaches $40 \%$, the retained Marshall stability of HRAM is close to the required value, while the freeze-thaw tensile strength is lower than the required value. When the RAP content reaches $50 \%$, both the retained Marshall stability and the freeze-thaw tensile strength of the the WRAM are close to the required values, indicating that the RAP content of the HRAM and the WRAM should be kept under $40 \%$ and $50 \%$, respectively.

\subsection{Low temperature crack resistance performance}

The test results are shown in Fig. 4.
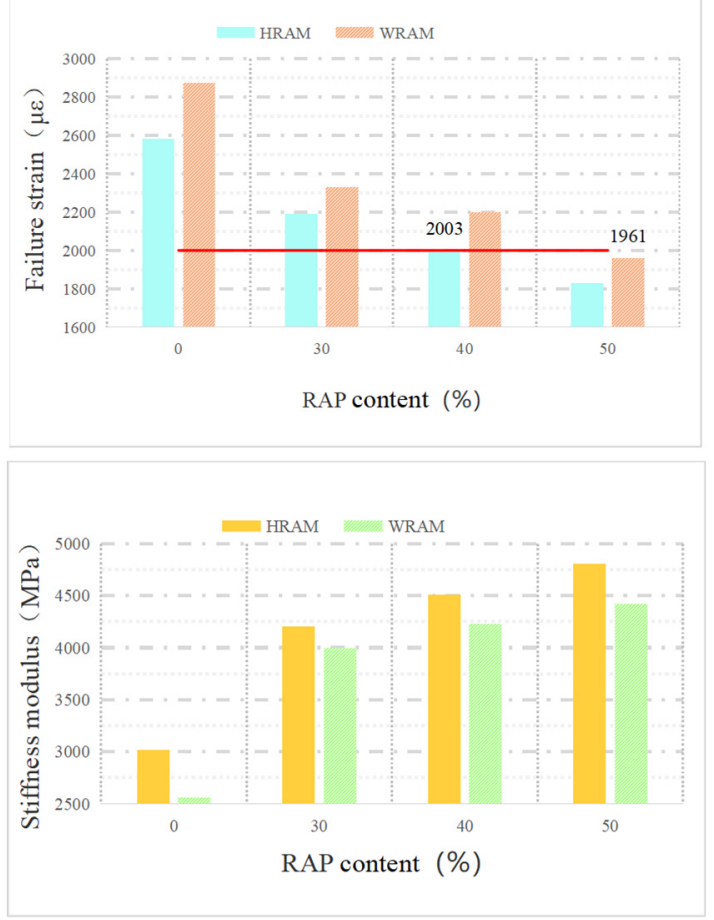

Fig. 4 Asphalt mixture beam bending test results

1). It can be drawn from Fig. 4, with the increase in RAP content, the failure strain of the WRAM and the HRAM decreases and the stiffness modulus increases, indicating the low temperature crack resistance performance of the WRAM and the HRAM lowers as RAP content increases.

2). Conditional on the same RAP content, the WRAM features better low temperature crack resistance than the HRAM.

3). When the content reaches $40 \%$, the failure value of the HRAM is close to the required value; when the content reaches $50 \%$, the failure strain of the WRAM is lower than the required value, which is because the asphalt in the RAP ages, and the adhesion is increasing and has poor low temperature performance. Thus the content of RAP in the HRAM and the WRAM should not exceed $40 \%$ and $50 \%$, respectively.

\section{Conclusion}

1). Conditional on $2.5 \%$ of water consumption, the expansion ratio and the half-life period can reach a good balance for the foamed asphalt to obtain good foaming performance. 
2). The physical foaming WRAM features better high temperature stability, water stability and low temperature crack resistance compared to HRAM.

3). The dynamic stability of the physical foaming WRAM and HRAM both increases notably as the RAP content increases, indicating that the increase in RAP content can significantly improve the high temperature stability of the recycled asphalt mixture.

4). The water stability and low temperature crack resistance of the physical foaming WRAM and HRAM drop as the RAP content increases. Therefore, in order to ensure good water stability and low temperature crack resistance, the RAP content in the physical foaming WRAM should be kept under 50\% and the RAP content in the HRAM should be kept under $40 \%$. The physical foaming warm-mix recycling technology can effectively enhance the RAP content ratio.

\section{Reference}

1. HE Liu. Discussion on construction technology and quality control of heat reclaimed green mixture in factory $[\mathrm{J}]$. Technology of highway and transport, 2016, 32 (2): 43-45.

2. WANG Jing. The study on application of SMA-13 hot mix plant recycling as the top layer[D]. Nanjing: Southeast University, 2017.

3. JI Jie, SUO Zhi, XU YING, et al. Experimental research on performance of warm-recycled mixture asphalt with SMA[J]. China Journal of Highway and Transport, 2013, 26(5):28-33.
4. HUANG Kangxu, LIANG Xingmin, XU Bo. Influences of RAP dosage on road performances of foamed warm mix reclaimed asphalt[J]. Journal of Materials Science and Engineering, 2016, 34(5): 794797.

5. GUO Naisheng, TAN Yiqiu, ZHAO Yinghua. Effect of key factors on performance of WMA containing RAP material[J]. Journal of Highway and Transportation Research and Development, 2015, 32(2):1-7.

6. HANG Yongqiang, CHENG Peifeng. Influence of RAP quantity on performance of warm mix recycled asphalt mixture $[\mathrm{J}]$. Journal of Highway and Transportation Research and Development, 2015, 32(12): 38-41.

7. TANG Wen, SHENG Xiaojun, XIE Xufei,et al. Performance of warm mix asphalt mixture containing different RAP contents[J]. Journal of Building Materials, 2016, 19(01):204-208.

8. XIONG Wentao, LIANG Yanfeng, XIAO Zhengjiang. Design and performance study on high warm mixing regeneration asphalt content mixture [J] Petroleum Asphalt, 2020, 34(2):26 32.

9. XIAO Peng, KANG Aihong, ZHONG Xingquan, et al. Study on road performance of foam-mixed reclaimed asphalt mixture $[\mathrm{J}]$. Petroleum Asphalt, 2018, 32(5): 8-12.

10. WANG Weiying. Performance Study on warm mix recycled asphalt binders and mixtures containing High percentages of RAP [D]. Dalian: Dalian University of Technology, 2018. 